\title{
BMJ Open Mapping registered nurse anaesthetists' intraoperative work: tasks, multitasking, interruptions and their causes, and interactions: a prospective observational study
}

To cite: Olin K, Göras C, Nilsson U, et al. Mapping registered nurse anaesthetists' intraoperative work: tasks, multitasking, interruptions and their causes, and interactions: a prospective observational study. BMJ Open 2022;12:e052283. doi:10.1136/ bmjopen-2021-052283

- Prepublication history and additional supplemental material for this paper are available online. To view these files, please visit the journal online (http://dx.doi.org/10.1136/ bmjopen-2021-052283)

Received 13 April 2021 Accepted 05 January 2022

Check for updates

(C) Author(s) (or their employer(s)) 2022. Re-use permitted under CC BY-NC. No commercial re-use. See rights and permissions. Published by BMJ.

For numbered affiliations see end of article.

Correspondence to

Karolina Olin;

karolina.olin@ki.se

\section{ABSTRACT}

Introduction Safe anaesthesia care is a fundamental part of healthcare. In a previous study, registered nurse anaesthetists (RNAs) had the highest task frequency, with the largest amount of multitasking and interruptions among all professionals working in a surgical team. There is a lack of knowledge on how these factors are distributed during the intraoperative anaesthesia care process, and what implications they might have on safety and quality of care.

Objective To map the RNAs' work as done in practice, including tasks, multitasking, interruptions and their causes, and interactions, during all phases of the intraoperative anaesthesia work process.

Methods Structured observations of RNAs $(n=8)$ conducted during 30 procedures lasting a total of 73 hours in an operating department at a county hospital in Sweden, using the Work Observation Method By Activity Timing tool.

Results High task intensity and multitasking were revealed during preparation for anaesthesia induction (79 tasks/hour, $61.9 \%$ of task time spent multitasking), anaesthesia induction (98 tasks/hour, 50.7\%) and preparation for anaesthesia maintenance (86 tasks/ hour, $80.2 \%$ ). Frequent interruptions took place during preoperative preparation (4.7/hour), anaesthesia induction (6.2 /hour) and preparation for anaesthesia maintenance (4.3 /hour). The interruptions were most often related to medication care $(n=54,19.8 \%)$, equipment issues $(n=40$, $14.7 \%)$ or the procedure itself $(n=39,14.3 \%)$. RNAs' work was conducted mostly independently $(58.4 \%)$, but RNAs interacted with multiple professionals in and outside the operating room during anaesthesia.

Conclusion The tasks, multitasking, interruptions and their causes, and interactions during different phases illustrated the RNAs' work as done, as part of a complex adaptive system. Management of safety in the most intense phases-preparing for anaesthesia induction, induction and preparing for anaesthesia maintenanceshould be investigated further. The complexity and adaptivity of the nature of RNAs' work should be taken into consideration in future management, development, research and education.

\section{Strengths and limitations of this study}

- This study adds to the limited knowledge on registered nurse anaesthetists (RNAs') intraoperative work, including tasks, multitasking, interruptions and their causes, and interactions, illuminating the changing intensity across different phases of intraoperative anaesthesia care.

- The data collection tool used, Work Observation Method By Activity Timing, employs a structured observation protocol with an operationalised definition of 'interruption', which may reduce the risk of potential measurement errors.

- Some participants were observed on several occasions, which may imply a risk of systematic bias.

- This study was performed at one hospital only, the observations did not include night shifts, weekend shifts or procedures conducted on Fridays, and the number of observed RNAs was relatively small, which may limit the representativeness and reduce the generalisability of the findings.

- It should be acknowledged that as the phases were constructed after the original data collection, some tasks, with long duration may extend across two or more phases, such as supervision, making the frequencies and proportion of times an estimate.

\section{INTRODUCTION}

Safe anaesthesia care is a fundamental, indivisible and indispensable part of healthcare delivery. ${ }^{1}$ The anaesthesia care has evolved into a highly technical and cognitively demanding care process, conducted in the rapidly changing, complex adaptive system (CAS) of an operating room (OR). ${ }^{2-4}$ The intraoperative anaesthesia work process consists of several phases: preoperative preparation, intraoperative care, induction of anaesthesia, maintenance of anaesthesia, emergence from anaesthesia, preparation for the postoperative period and extubation. ${ }^{56}$ 
The WHO's surgical safety checklist, including sign in, time out and sign out, should be taken into account during work. ${ }^{7}$ Phases and tasks have been mapped in flow charts ${ }^{8}$ and described generally, with a focus on ergonomics and human factors, ${ }^{2}{ }^{6-12}$ efficiency of the processes ${ }^{13} 14$ and on how safety emerges using systemic approach. ${ }^{15} 16$ According to resilience engineering, healthcare and resilience can be defined as the ability of the CAS to rapidly respond and adapt to both expected and unexpected conditions. ${ }^{17} \mathrm{~A}$ way to learn from such adaptations may be through identifying differences between work as planned by those who write the procedures and work as done in practice. ${ }^{17}$ This has led to a focus on understanding and describing everyday clinical work processes and tasks. ${ }^{18}$ The aim of this study is to broaden this understanding through elucidating registered nurse anaesthetists (RNA's) work as done.

Multitasking-managing multiple tasks simultaneously ${ }^{19}$-is one adaptation that has been studied in acute care at emergency departments, ${ }^{20-24}$ intensive care units ${ }^{25}$ and in ORs, from the perspectives of surgeons ${ }^{26}$ and surgical teams. ${ }^{27}$ Multitasking is often used as a strategy to cope with increased work and task density, ${ }^{23}$ and has been described as an integral and appropriate part of acute care. ${ }^{22}{ }^{23}$ Multitasking has also been identified as a factor related to errors and adverse events, especially when there is a high cognitive load. ${ }^{2324}$ However, in order to be able to prevent errors, it is important to map the prevalence of multitasking and the situations when it occurs during RNAs' intraoperative work.

Serious adverse events are relatively rare in anaesthesia, but may cause considerable harm or even death. ${ }^{28}$ However, interruptions and disturbances are present in every operation. ${ }^{29}{ }^{30}$ As a phenomenon, interruptions, disruptions and distractions have been challenging to encapsulate, resulting in multiple overlapping definitions. Interruption has been defined as 'discontinuity in task performance, an intrusion of a secondary, unplanned task and externally or internally initiated '. ${ }^{31}$ Interruptions have an inherent potential to cause harm if they affect the progress of safe care by causing lapses in attention, delays of tasks, or unnecessary multitasking. ${ }^{32}$ It has been acknowledged that interruptions may also be a strategy for a team to handle complexity and ensure patient safety, for example, when raising concerns about a possible risk. ${ }^{2732} 33$ Although adaptive responses to interruptions and multitasking in interaction with the surgical team may be key to safety performance in anaesthesia care, ${ }^{27}$ interruptions and distractions remain a safety risk and need to be investigated further. ${ }^{31}$

In the OR context, interruptions have been investigated from the perspectives of the surgical team ${ }^{27} 34$ and of the surgeon. ${ }^{356}$ In anaesthesia care, interruptions have been investigated focusing on anaesthesiologists' work during procedures, ${ }^{37} 38$ during specific phases of the anaesthesia process, such as during preoperative care ${ }^{29}$ or induction, ${ }^{32} 3940$ or in simulated environment. ${ }^{41}$ In anaesthesiologists' work certain moments of the anaesthesia process involving task complexity and workload, such as emergence for anaesthesia, were identified as more prone to being affected. ${ }^{11}{ }^{41}$ Self-initiated, non-clinical distractions such as reading or listening to the radio were found to be common among anaesthesiologists and RNAs, without impairing vigilance or causing adverse events. ${ }^{42}$ In addition, external staff, opening doors, handling telephones and equipment-related and procedure-related issues may cause intraoperative interruptions. ${ }^{27} 34$ Thus, in order to plan effective interventions for management of interruptions, the risk-prone moments in the real work processes of the RNAs should be explored.

The professional roles of RNAs' vary between countries, ${ }^{43}$ making direct comparison of studies on intraoperative anaesthesia care challenging. RNAs' work has been studied as regards to non-technical skills, ${ }^{445}$ experiences from clinical work processes, ${ }^{46}{ }^{47}$ skills assessment using simulation, ${ }^{48}$ experiences of the WHO's surgical safety checklist, ${ }^{49}$ patient advocacy ${ }^{50} 51$ and work experiences. ${ }^{52}$ Among the professionals working in the surgical team, RNAs had the highest task frequency, with the largest amount of multitasking and interruptions. ${ }^{27}$ However, there is a lack of knowledge on how these factors are distributed during the intraoperative anaesthesia care process, and what implications they might have for safety and quality of care. To conclude, RNAs' intraoperative anaesthesia work process has not yet been described, which makes elaboration of potentially risk-prone moments and their management in practice challenging. The aim of this study was to map the RNAs' work in practice, including tasks, multitasking, interruptions and their causes, and interactions during all phases of the intraoperative anaesthesia work process.

\section{METHODS}

\section{Study design and setting}

This prospective observational study mapped RNAs' intraoperative anaesthesia work process. This study was part of a structured direct observational time and motion study of operating teams (surgeons, OR nurses and RNAs), which was conducted in an OR department at a local county hospital in Sweden. ${ }^{27}$ The hospital had three surgical inpatient wards (62 beds in total), performing about 4100 surgeries annually. The central OR department had six rooms that served both acute and elective orthopaedic and surgical patients. Each operation room had a separate preparation room, with anaesthesia equipment, medication and equipment for monitoring the patient.

\section{Sample}

The unit of observation in this study was the RNAs' intraoperative work process. The rest of the surgical team was captured indirectly through documentation of tasks, including interactions with other team members or external professionals. No patients were observed. Observed procedures were selected from the case list in 
the OR department, based on a convenience sample. The observation sessions were selected to achieve coverage across weekdays (Monday-Thursday) and times (07:3021:00 hours). To capture variation, different surgical procedures were chosen and both acute and elective surgical procedures performed in adults were included. Observations were not conducted in ORs with orthopaedic procedures, due to infection control regulations in the hospital. ${ }^{43}$

In Sweden, RNAs are qualified nurses with a graduate diploma in specialist nursing, who independently induce, maintain and end general anaesthesia, including extubation, with support from anaesthesiologists, who are ultimately medically responsible. The anaesthesiologist's role depends on the patient's classification, as described by the American Society of Anesthesiologists, and the acuteness of the procedure. ${ }^{53}$ The role of an RNA is to safeguard the intraoperative care process for the patient. In total, 16 RNAs were eligible for participation, with eight being observed. On mean, the observed RNAs had 18 years' experience as specialists (range 3-28) and 14 years' experience as RNAs at the study hospital (5-34). Their mean age was 50 years (32-64). Three were women and five men.

The relatively small number of RNAs included was due to practicalities regarding possible observable procedures and the RNAs' working schedules, with all RNAs being observed more than once, between 2 and 6 times. Written informed consent was obtained from all observed RNAs prior to the procedures. In addition, patients were informed about the observations and were given the opportunity to opt out. However, all gave their verbal consent. The study was conducted in accordance with the Helsinki declaration of research ethics. ${ }^{54}$

\section{Instrument}

Observational data of the RNAs' work process was recorded on a portable touchscreen tablet (Lenovo 7 Tab3) running the Work Observation Method By Activity Timing (WOMBAT) software. ${ }^{55}$ The software takes into account multiple dimensions of work, as well as specific task categories and subcategories within these dimensions. Task categories were adapted to fit the RNAs' work tasks as described in a previous exploratory study (online supplemental appendix 1). ${ }^{27}$ Each task classification included information about the task activity undertaken (What), with whom (Who) the participant interacted (eg, other members of the surgical team), resources (How) used (eg, telephone) and the causes (Why) of any interruptions or multitasking that occurred. Multitasking and interruptions have been given different definitions, ${ }^{19} 56$ and in this study interruptions were defined as stopping a current task to respond to an external stimulus such as a pager, and multitasking was defined as performing two tasks simultaneously. Both were recorded in WOMBAT. Tasks performed by the RNAs and causes of interruptions (Why) were recorded based on the predefined categories (online supplemental appendix 2). Training and pilottesting were performed prior to actual observations.

Inter-rater reliability was calculated in accordance with the WOMBAT manual using a comparison between the observers' recordings as regards frequency of tasks. In total, 12 rounds of inter-rater reliability testing were conducted by the researchers, independently observing the same participants for $30 \mathrm{~min} .{ }^{27}$ Inter-rater reliability was calculated by comparing the numbers and types of tasks recorded by the two researchers. Cohen's kappa was calculated from the last three pilot observations. The most frequently observed tasks were 0.85 for indirect care (pre and intra), 0.87 for direct care, 0.93 for medication and 0.82 for communication. According to the WOMBAT manual, once an inter-rater reliability $\geq 0.81$ has been achieved, data collection may commence. The total Cohen's kappa score for frequency of tasks was 0.86 , which was regarded as a high observer agreement. ${ }^{57}$

\section{Data collection}

Two researchers (CG and KO) performed the observations of the surgical teams, between 07:30 and 21:00 hours on weekdays from 14 November 2016 to 15 December 2016, resulting in a database of 11791 tasks. In an earlier publication, we have presented the overall findings at a team level, covering 26 observed procedures per profession. ${ }^{27}$ However, RNAs were observed during 30 procedures, which is the sample used in this study. The observations started when an RNA began the planning and preparation for a patient's anaesthesia in the OR and stopped when the patient was transported from the OR to the recovery room. Each participating RNA was shadowed unobtrusively. None of the observations exceeded 4 hours in length, because the researchers' ability to concentrate and the quality of observations might have been affected by longer observation periods.

\section{Data analysis}

All data containing observations of RNAs' tasks, multitasking, interruptions and their causes, and interactions were separated from the original database by the first observer (KO), resulting in a dataset with 5291 tasks in total. In order to describe the work done by RNAs in the intraoperative anaesthesia work process in greater detail, the observational data with RNAs' tasks were grouped based on the previously identified phases of anaesthesia care ${ }^{5-7}$ using the start time of tasks in each phase. In addition, recurring work patterns were identified, such as preparation for induction and maintenance of anaesthesia. These were included in the intraoperative anaesthesia work process (online supplemental appendix 3), which was then used as a framework for sorting the data regarding tasks, multitasking, interruptions and interactions with other professionals in or outside the OR. The second observer (CG) confirmed the sorting of the data and phase-specific outcomes.

Quantitative analysis of data was performed using Microsoft Excel (2016) and the Statistical Package for 
Social Sciences (IBM SPSS Statistics; V.21). To analyse work patterns in the anaesthesia process, descriptive statistics were used, including total observation time, number of tasks, task frequency, total task time (hours), categoryspecific task time, proportion of category-specific task time, category-specific multitasking time, proportion of task time spent multitasking, interruption rate per hour and number of causes of interruptions.

\section{RESULTS}

\section{Demographics}

In total, 30 procedures were observed, extending across 73 hours, starting when a RNA entered the OR and began to plan and prepare for the anaesthesia, and lasting until the patient was transported from the OR to a recovery area. Time from incision until end of surgical procedure ranged between $38 \mathrm{~min}$ and 3 hours and $15 \mathrm{~min}$. Out of the 30 procedures, two were acute and 28 elective, with 29 performed under general anaesthesia and one under spinal anaesthesia. In 12 procedures, a nursing student was being supervised.

\section{Observed tasks, multitasking, interruptions and cooperation partners per phase}

A total of 5291 tasks (72.5 tasks/hour) were identified in the RNAs' intraoperative anaesthesia work process. Communication was the most frequently observed task $(\mathrm{n}=1264,23.9 \%$, out of which conducting phone calls $\mathrm{n}=160)$ with intraindirect care as the second $(\mathrm{n}=1188$, $22.5 \%$ ) (table 1). A detailed description of task and subcategories can be seen in online supplemental appendix 1 .

During the intraoperative anaesthesia work process, RNAs spent $62.3 \%$ of their time multitasking. The proportion of time spent multitasking was highest during preparation for anaesthesia maintenance $(80.2 \%)$ and before initiation of surgery. During preparation for anaesthesia induction and in maintenance of anaesthesia, the RNAs multitasked more than half of the time $(61.9 \%$ and $63.5 \%$, respectively). The only phase in which multitasking was not highly prevalent was preoperative preparation (table 2).

However, looking at category-specific task time, the highest proportion of time was spent on intraindirect care (52 hours $55 \mathrm{~min}, 41.9 \%$ ), followed by supervision (23 hours $11 \mathrm{~min}, 18.4 \%$ ) and direct care (12 hours 16 $\min , 11.7 \%$ ). The distribution of tasks varied between phases, reflecting the changing demands in the intraoperative anaesthesia work process (figure 1). RNAs spent a lot of time on supervision at the beginning of the procedures, such as during preoperative preparation and during preparation for anaesthesia induction. Direct care was apparent during preparation, induction and extubation. The rest of the anaesthesia time was indirect care.

Interruptions occurred most frequently during anaesthesia induction (6.2/hour), during preoperative preparation (4.7/hour) and during preparation for anaesthesia maintenance (4.3 /hour) (figure 2). In all,
272 interruptions were identified (3.7/hour). The interruptions in RNAs' work most often involved medication care $(n=54,19.8 \%)$, equipment-related issues $(n=40$, $14.7 \%)$, such as missing or malfunctioning equipment, or were procedure-related $(n=39,14.3 \%)$, such as the surgeon asking for a rapid change of patient's position. RNAs reacted to interruptions primarily by professional communication $(n=51,23.1 \%)$ and by medication carerelated activities $(n=41,18.6 \%)$, such as administering medication.

Most of the RNAs' time in the OR was spent on independent work (76 hours $20 \mathrm{~min}, 58.4 \%$ ) (figure 3). Time was also spent in interaction with a student (19 hours $51 \mathrm{~min}, 15.2 \%$ ) or directly with the patient (19 hours $05 \mathrm{~min}, 14.6 \%$ ), the circulating nurse ( 3 hours $48 \mathrm{~min}$, $2.9 \%$ ) or the anaesthesiologist (2 hours $46 \mathrm{~min}, 2.1 \%$ ). Most interactions took place during preparation for anaesthesia induction, induction, preparation for extubation or extubation.

\section{DISCUSSION}

This study contributes with new insights into how RNAs' tasks, multitasking, interruptions and their causes, and interactions are distributed during the intraoperative anaesthesia work process. In Sweden RNAs' role is relatively independent, and their responsibilities cover most of the intraoperative anaesthesia care process, making them a key contributor to intraoperative safety. ${ }^{53}$ Based on the observations, the task frequency $(72.5$ /hour), proportion of multitasking $(62.3 \%)$ and interruptions ( $\mathrm{n}=272,3.7$ /hour) cumulated often simultaneously in certain phases, such as during anaesthesia induction, preparation for anaesthesia maintenance and extubation. RNAs conducted most of their intraoperative anaesthesia work independently $(58.4 \%)$, but when interacting, it involved multiple professionals, also outside the OR. When combining these different aspects of practice, a picture of a complex and dynamic work process is revealed. Previous research regarding RNAs' work has focused on areas such as non-technical skills, ${ }^{44} 45$ experiences from different clinical aspects of work ${ }^{46-49}$ patient advocacy ${ }^{50}$ and work experiences. ${ }^{52}$ This study builds on this knowledge by illuminating how RNAs' work is actually done, uniquely mapping the different dimensions of work in relation to the intraoperative work process and thus providing further insights into the context and challenges for safe practice. As the core of anaesthesia care and the safety-critical moments are the same regardless of context, this study presents possibilities for both training-timing the use of non-technical skills to safetycritical moments-and for design of safer work practices in relation to the different intraoperative phases. Below, the findings are presented linked to the phases of the anaesthesia work process.

\section{Preparation}

During preoperative preparation, that is, when starting the work process in the OR, multitasking was not prevalent. 


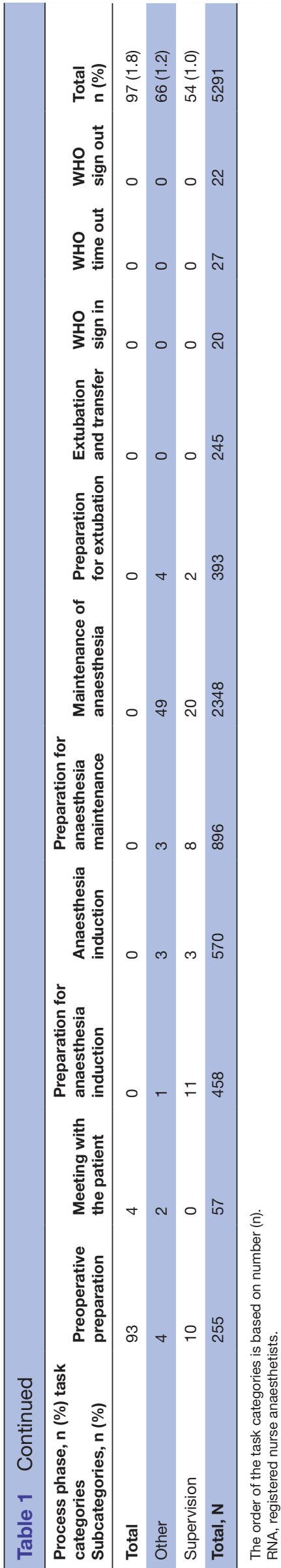

Observable interruptions were common, and RNA left the OR to retrieve necessary equipment and medications, prior to the patient's arrival. Interruptions may thus be expected and even necessary, rather than unwanted, as they were related to the task at hand. ${ }^{32}$ Preparation before anaesthesia through organising the work environment, creating a plan for the patient and undergoing mental preparation may be a way to decrease the number of unnecessary interruptions during anaesthesia. This may contribute to creating safe care through increased readiness to respond to both expected and unexpected events during intraoperative anaesthesia care. ${ }^{47}$ If no nursing student was present and supervision was not required, the preoperative preparation was mainly performed independently, which could explain the rarity of multitasking. Given the cognitive load, it could be beneficial to assign the supervision of students to experienced staff, for whom multitasking may be easier to manage. ${ }^{58}$

Preparation for anaesthesia induction was prone to high task intensity and multitasking. There were somewhat fewer interruptions than during preoperative preparation, but a lot of interaction with other members of the team and with the patient. Successful teamwork has a major impact on patient safety, requiring coordinating, reaffirming ${ }^{47}$ and assertive communication when sharing information. ${ }^{59}$ RNAs' ability to adaptively coordinate their activities during routine situations as a part of the surgical team may support the management of non-routine events. ${ }^{60}$ Non-technical skills, such as task management, team working, situational awareness and decision making have been identified as prerequisites for sustaining safe task performance. ${ }^{44}$ Hence, in RNAs' work, non-technical skills could support the management of phases with high task frequency, multitasking and interruptions. ${ }^{61}$

\section{Anaesthesia induction}

Anaesthesia induction has been identified as a safetycritical phase. ${ }^{2940}$ In this study, the highest task frequency for RNAs was during anaesthesia induction. The same phenomenon has previously been observed with anaesthesiologists in simulated situations, ${ }^{26962}$ confirming that induction may be equally intensive for the entire anaesthesia team. In this study, the frequency of interruptions was high during anaesthesia induction. RNAs' work was ceased because of equipment-related and procedurerelated issues. In addition, phone calls were answered, which are known cause a high level of disturbance in terms of consequences and duration. ${ }^{32}$ Interruptions may present a threat to situational awareness and prospective memory, both of which are required when continuously monitoring tasks, detecting changes and sharing information with the a team. ${ }^{63}$

Interruptions during induction have been reported in previous studies on RNAs and anaesthesiologists as a team $^{32}$ and on anaesthesiologists. ${ }^{29}{ }^{38}$ However, in other studies, ${ }^{3841}$ the most interrupted phase of anaesthesia was emergence, not induction. In these studies, induction occurred in a separate induction room, while in our study 
Table 2 Frequency of tasks, total multitasking time and multitasking as a proportion of the total phase-specific observation time in RNAs' intraoperative work process

\begin{tabular}{|c|c|c|c|c|}
\hline $\begin{array}{l}\text { Phases of RNAs' intraoperative } \\
\text { anaesthesia work process }\end{array}$ & $\begin{array}{l}\text { Total phase-specific } \\
\text { observation time, hours } \\
\text { and minutes }\end{array}$ & $\begin{array}{l}\text { Frequency of } \\
\text { tasks } \\
\text { (n/hrs) }\end{array}$ & $\begin{array}{l}\text { Total multitasking } \\
\text { time, hours and } \\
\text { minutes }\end{array}$ & $\begin{array}{l}\text { Multitasking time as a } \\
\text { proportion of total phase- } \\
\text { specific observation time }\end{array}$ \\
\hline Preoperative preparation & $4: 50$ & 53 & $1: 21$ & $27.9 \%$ \\
\hline Meeting with the patient & $1: 15$ & 47 & 0 & 0 \\
\hline Preparation for anaesthesia induction & $5: 49$ & 79 & $3: 36$ & $61.9 \%$ \\
\hline Anaesthesia induction & $5: 49$ & 98 & $2: 57$ & $50.7 \%$ \\
\hline Preparation for anaesthesia maintenance & $10: 25$ & 86 & $8: 21$ & $80.2 \%$ \\
\hline Maintenance of anaesthesia & $36: 02$ & 65 & $22: 53$ & $63.5 \%$ \\
\hline Preparation for extubation & $4: 48$ & 85 & $2: 56$ & $43.1 \%$ \\
\hline Extubation and transfer & $3: 15$ & 79 & $1: 55$ & $60.0 \%$ \\
\hline WHO Sign in & $0: 14$ & * & $0: 08$ & $57.1 \%$ \\
\hline WHO Time out & $0: 28$ & * & $0: 17$ & $60.1 \%$ \\
\hline WHO Sign out & $0: 13$ & * & $0: 07$ & $53.8 \%$ \\
\hline Total & $72: 59$ & 72 & $45: 23$ & $62.3 \%$ \\
\hline
\end{tabular}

*Not possible to calculate.

RNA, registered nurse anaesthetists.

the patient was already in the OR when anaesthesia was administered. This highlights the impact of the work environment and its design on patient safety. So-called 'sterile cockpit thinking' has been suggested as an alternative when a separate room is not an option. ${ }^{64}$ This means that the entire surgical team has to acknowledge and respect other team members' work phases, when interruptions and unnecessary multitasking could cause risks and deviations to a patient's care process. 394165

\section{Anaesthesia maintenance}

For the most part, RNAs independently conducted the preparation for anaesthesia maintenance. Both the task frequency and the interruption frequency were high, as

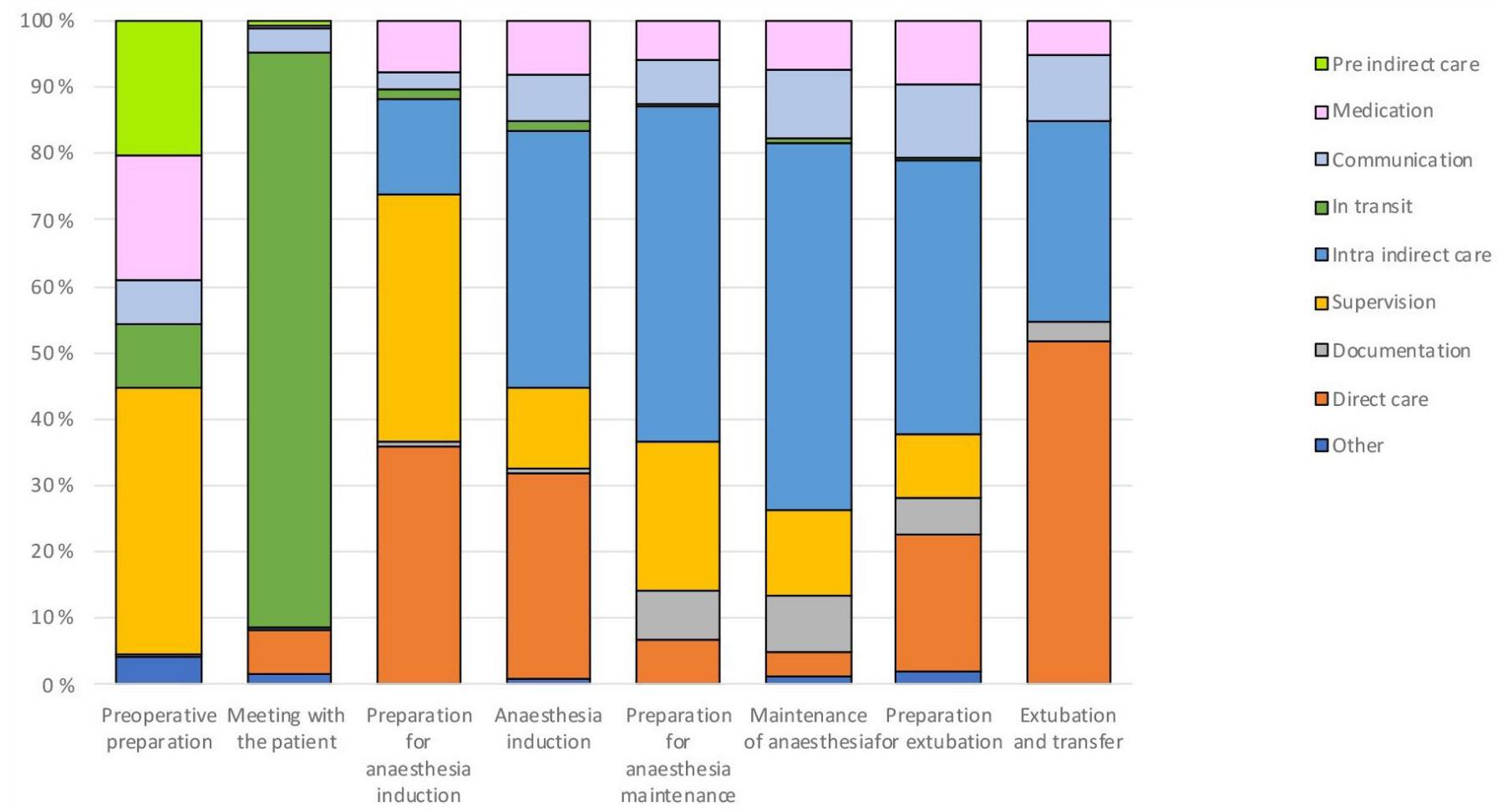

Figure 1 Proportions of category-specific task times in the RNAs' intraoperative anaesthesia work process. RNA, registered nurse anaesthetists. 


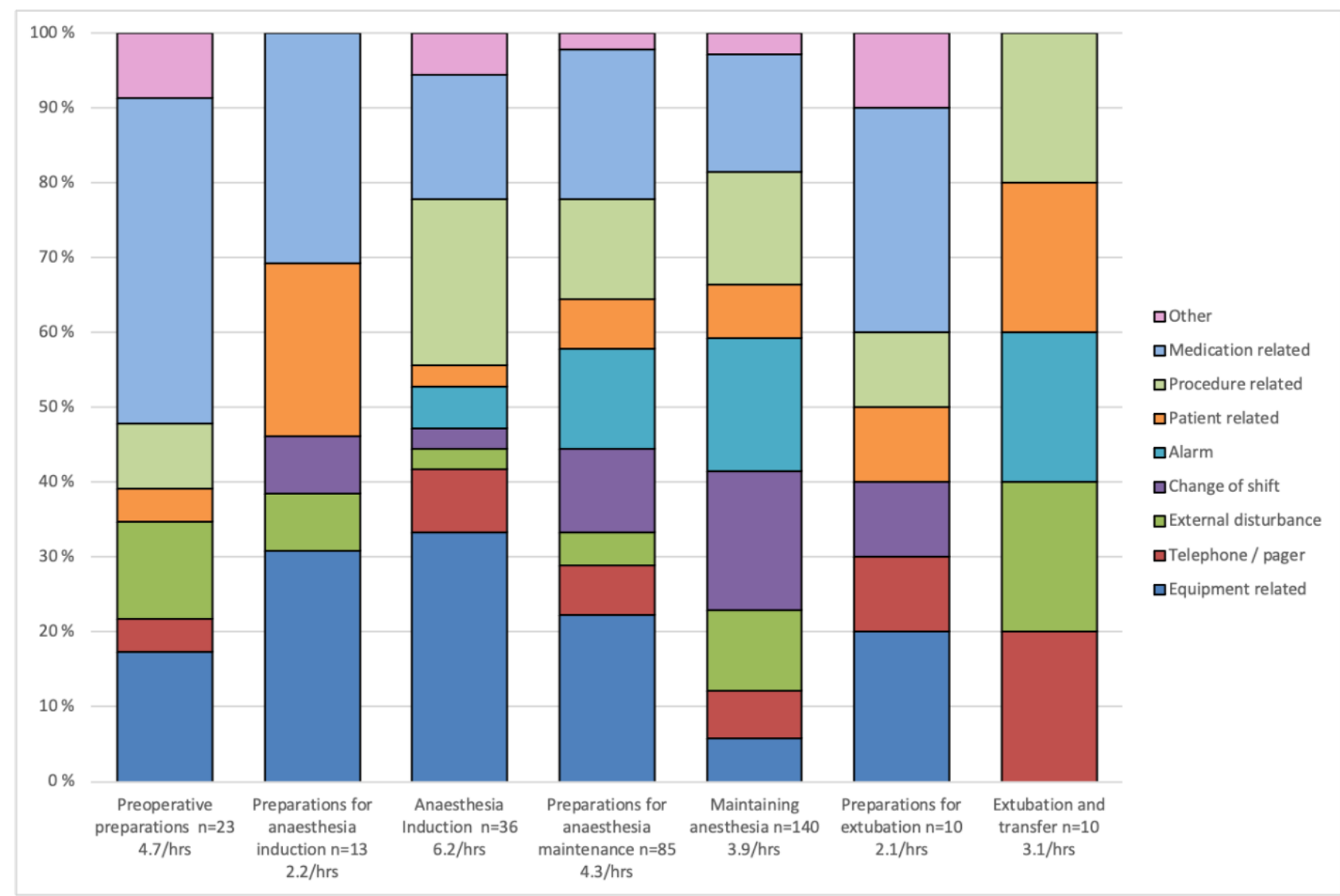

Figure 2 Proportions, number and frequencies of causes of interruptions in the phases of the RNAs' intraoperative anaesthesia work process. Note-no interruptions were observed during the phase 'meeting the patient', hence it is not included in the figure. RNA, registered nurse anaesthetists.

also identified in Al-Hakim et al; this phase also had the highest proportion of multitasking. ${ }^{29}$ This may reflect the requirements of the phase, where tasks need to be performed immediately and cannot be delayed. Multitasking can also be a consequence of interruptions ${ }^{29}$ or may reflect the need to perform more effectively during a shorter period of time, due to increased production pressure. ${ }^{30}$ If avoidable and unnecessary interruptions could be minimised, the surgical team could have a greater cognitive capacity to respond to complexities when needed. ${ }^{29}$

\section{Extubation}

During preparation for extubation, the frequency of tasks was elevated again, and the amount of multitasking was also high. In contrast to this study, emergence was the most disturbed phase in a study by Campbell $e t a l,{ }^{38}$ with the noise level being significantly higher during emergence than during induction. ${ }^{38}$ Extubation has been identified as a safety-critical phase, ${ }^{38}{ }^{41}$ during which RNAs need to be a step ahead and the rest of the surgical team should respect the importance of the moment. ${ }^{46}$ Thus, sterile cockpit thinking ${ }^{41}$ could be beneficial also at the end of anaesthesia. The need of RNAs to focus on the safetycritical task at hand could be highlighted outside the OR too, possibly with the help of IT solutions. This could be achieved with a screen showing the work phases. Patient safety could be enhanced by marking the start and end of a critical phase, during which interruptions should be avoided and no one should enter the OR without a valid reason.

\section{Coordination of the anaesthesia care process}

In this study, RNAs communicated often and using short time frames, with professionals in OR and with external units, reflecting their coordinating role in surgical team. This could indicate an active approach to managing the complex everyday work, which requires anticipating, responding, monitoring and adapting based on the needs of the team and the patient. ${ }^{66}$ Conducting phone calls $(n=116)$ as a part of professional communication may be imperative for the smooth delivery of the perioperative process and enhance preparedness for the next phase in the process. Another sign of coordination was the brief moments RNA needed to leave the OR in order to retrieve something missing from the preparation room. These in transit-tasks $(n=123)$ appeared in every phase, including anaesthesia induction. However, especially during a safety-critical or task-intensive phase, such as anaesthesia induction, preparation for maintenance, extubation or an unexpected acute situation, calls and retrieval of medication or equipment needed could be 


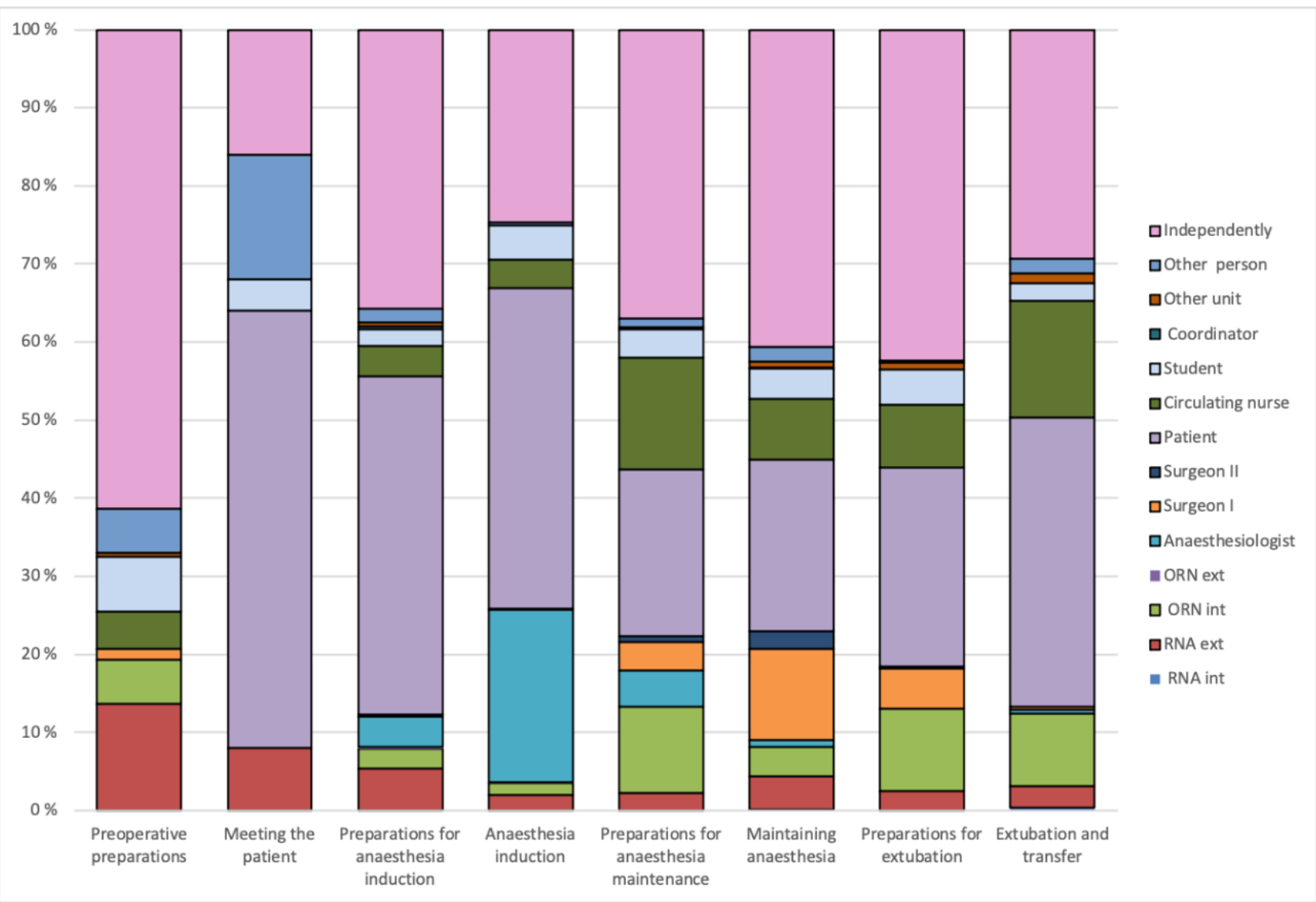

Figure 3 Proportions of time that RNAs spent in independent work and in interaction with other professionals and patients during the phases of the intraoperative anaesthesia work process. RNA, registered nurse anaesthetists.

delegated to other members of the surgical team, such as the circulating nurse. In addition, thorough preparations before anaesthesia may ensure the adequate amount of necessary appliances in the OR.

\section{The dual nature of multitasking}

Multitasking is an inherent part of intraoperative anaesthesia care and should be embraced as such. Some professionals view multitasking as a professional skill, not as a strain. ${ }^{21}$ Successful management of multitasking requires professional experience ${ }^{67}$ and the ability to adapt to rapidly changing situations while simultaneously ensuring the seamless progress of anaesthesia care. However, recent research on the effects of multitasking ${ }^{67}$ shows that it increases complexity and cognitive load, and the inherent risks should be acknowledged. Unnecessary multitasking should be avoided, especially during safetycritical phases such as anaesthesia induction and extubation. Management could use assessment of the amount and quality of multitasking as a leading indicator of potential safety issues, when work actually done in anaesthesia is drifting too far from work as planned.

\section{Management}

RNAs have a pivotal role in managing the complexity in a CAS and sustaining patient safety together with the surgical team. However, managerial decisions and culture at the organisational level have implications on the complexity at the sharp end, where work is actually done, as well as effects on the adaptive capacity of the frontline. ${ }^{68}$ High demands on production or time pressure may increase the workload and push professionals to undertake avoidable multitasking. Our study shows that there are aspects of the RNAs' contributions to resilient performance of surgical team tasks that could be harnessed by observing the natural, case-related variation of tasks, multitasking and interruptions. This could aid in organising healthcare to support resilience. However, we have also described examples of how the RNAs' attention can be consumed by adaptations needed to cover the consequences and unnecessary complexity of a poorly designed system. ${ }^{63}$ Detailed description and analysis of tasks, multitasking, interruptions and their causes, and interactions during the different phases in the RNAs' work process may provide insight into risk-prone situations. This could elucidate options for organising healthcare to close the gap between the work as planned and the work as done and thus protect the capacity of RNAs to contribute to resilient performance.

\section{Strengths and limitations}

This study adds to the knowledge on RNAs' intraoperative work, including tasks, multitasking, interruptions and 
their causes, and interactions, illuminating the varying intensity across different phases of intraoperative anaesthesia care. The data collection tool used, WOMBAT, employs a structured observation protocol with an operationalised definition of 'interruption', which may reduce the risk of potential measurement errors. However, the number of RNAs observed was relatively small, limiting the representativeness. In addition, some participants were observed on several occasions, which may imply a risk of systematic bias. On the other hand, the unit of observation was the RNAs' work process, which depended additionally on the ongoing operation and the operating environment. Generally, this could decrease the impact of the individual nurses observed. This study was performed at one hospital only and the observations did not include night shifts, weekend shifts or procedures conducted on Fridays, which may limit the representativeness for different work shifts and reduce the generalisability of the findings. It should be acknowledged that as the phases were constructed after the original data collection; some tasks with long duration may extend across two or more phases, making the frequencies and proportion of times an estimate. This was especially evident with respect to supervision, as this continued throughout the entire intraoperative anaesthesia work process.

\section{CONCLUSION}

This study adds to the knowledge from previous research by illuminating how RNAs' work is actually done, uniquely mapping the different dimensions of work in relation to the intraoperative work process and thus giving further insights into the context and challenges for safe practice. As the core of anaesthesia care and thus the safetycritical moments are the same regardless of context, this study presents possibilities for learning regarding how to reduce the occurrence of unnecessary interruptions and disturbances in RNAs' intraoperative work. Phases more prone to unnecessary multitasking and interruptions, especially those that are critical for the patient and cognitively demanding for the RNAs, should be acknowledged and their safety ensured. This should be taken into consideration already in planning the processes, taking account of the management, surgical team, work environment and available resources. The results can also contribute to development of training by elucidating safety-critical moments, threatened by untimely multitasking or interruptions. This knowledge suggests specific timing for the use of non-technical skills and could also guide the design of safer work practices specifically tailored to safetycritical intraoperative phases. Multitasking and interruptions being seen as threats to safety in anaesthesia care, they could be interpreted as signs of the adaptive capacity of a complex system, reflecting resilience. Further studies exploring this adaptive capacity of RNAs in managing the intraoperative anaesthesia work process, multitasking, interruptions, and interactions may give insights into how
RNAs could make note of and act on situations that introduce a risk.

Author affiliations

${ }^{1}$ Medical Management Centre, Department of Learning, Informatics, Management and Ethics, Karolinska Institute, Stockholm, Sweden

${ }^{2}$ Administration Centre, Tyks and Hospital District of Southwest Finland, Turku, Finland

${ }^{3}$ Faculty of Medicine, School of Education, Health and Social Studies, Örebro University, Orebro, Sweden

${ }^{4}$ Center for Clinical Research Dalarna, Falun, Sweden

${ }^{5}$ Department of Anaesthesia and Intensive Care Unit, Falu Hospital, Falun, Sweden

${ }^{6}$ Department of Neurobiology, Care Sciences and Society, Karolinska Institutet, Stockholm, Sweden

${ }^{7}$ School of Health and Welfare, Dalarna University, Falun, Sweden

${ }^{8}$ Astrid Lindgren's Children's Hospital, Karolinska Universitetssjukhuset, Stockholm, Sweden

${ }^{9}$ Department of Health and Caring Sciences, Faculty of Health and Life Sciences, Linnaeus University, Kalmar, Sweden

Acknowledgements We want to thank Mesfin Kassaye Tessma and Ville Pöntinen for statistical support. We also thank the heads of participating departments and the RNAs for their willingness to participate in this study.

Contributors CG, MU, UN, AE and ME conceptualised the design of the main project. KO, CG, KPH and ME were responsible for the design and methodology (mapping and visualisation of process phases) of the current study. CG was project administrator and performed the initial exploratory observations. CG and KO were responsible for the identification and definitions of categories, as well as data collection. KO curated the data, performed the statistical analysis, undertook the initial interpretation of data and was responsible for the original draft. CG, ME and $\mathrm{KPH}$ contributed with critical interpretation of data and substantial intellectual content to the manuscript. The manuscript was critically reviewed and edited by all authors. All authors have read and approved the final manuscript. KO is the guarantor.

Funding This work was funded by The Centre for Clinical Research Dalarna and through Finnish state funding for university-level health research.

Disclaimer The funders have neither been involved in any part of the study, nor in writing the manuscript or the decision to submit the manuscript for publication.

Competing interests None declared.

Patient consent for publication Not applicable.

Ethics approval Ethical approval was provided by the regional ethical review board in Uppsala, Sweden (reference number 2016/264).

Provenance and peer review Not commissioned; externally peer reviewed. Data availability statement № data are available.

Supplemental material This content has been supplied by the author(s). It has not been vetted by BMJ Publishing Group Limited (BMJ) and may not have been peer-reviewed. Any opinions or recommendations discussed are solely those of the author(s) and are not endorsed by BMJ. BMJ disclaims all liability and responsibility arising from any reliance placed on the content. Where the content includes any translated material, BMJ does not warrant the accuracy and reliability of the translations (including but not limited to local regulations, clinical guidelines, terminology, drug names and drug dosages), and is not responsible for any error and/or omissions arising from translation and adaptation or otherwise.

Open access This is an open access article distributed in accordance with the Creative Commons Attribution Non Commercial (CC BY-NC 4.0) license, which permits others to distribute, remix, adapt, build upon this work noncommercially, and license their derivative works on different terms, provided the original work is properly cited, appropriate credit is given, any changes made indicated, and the use is non-commercial. See: http://creativecommons.org/ licenses/by-nc/4.0/.

ORCID iDs

Karolina Olin http://orcid.org/0000-0002-7482-2950

Camilla Göras http://orcid.org/0000-0002-0883-4072

Mirjam Ekstedt http://orcid.org/0000-0002-4108-391X 


\section{REFERENCES}

1 Meara JG, Leather AJM, Hagander L, et al. Global surgery 2030: evidence and solutions for achieving health, welfare, and economic development. Lancet 2015;386:569-624.

2 Manser T, Wehner T. Analysing action sequences: variations in action density in the administration of anaesthesia. Cogn Technol Work 2002;4:71-81.

3 Nemeth C, O'Connor M, Klock PA, et al. Discovering healthcare cognition: the use of cognitive artifacts to reveal cognitive work. Organization Studies 2006;27:1011-35.

4 Mahajan A, Islam SD, Schwartz MJ, et al. A hospital is not just a factory, but a complex adaptive System-Implications for perioperative care. Anesth Analg 2017;125:333-41.

5 Tollerud L, Botsford J, Hoglan MA, et al. A model for perioperative nursing. Aorn J 1985;41:188-94.

6 Phipps D, Meakin GH, Beatty PCW, et al. Human factors in anaesthetic practice: insights from a task analysis. $\mathrm{Br} \mathrm{J}$ Anaesth 2008;100:333-43.

7 Haynes AB, Weiser TG, Berry WR, et al. A surgical safety checklist to reduce morbidity and mortality in a global population. $N$ Engl J Med Overseas Ed 2009;360:491-9.

8 Fowler $\mathrm{PH}$, Craig J, Fredendall LD, et al. Perioperative workflow: barriers to efficiency, risks, and satisfaction. Aorn $J$ 2008;87:187-208.

9 Manser T, Dieckmann P, Wehner T, et al. Comparison of anaesthetists' activity patterns in the operating room and during simulation. Ergonomics 2007:50:246-60.

10 Weinger MB, Herndon OW, Zornow MH, et al. An objective methodology for task analysis and workload assessment in anesthesia providers. Anesthesiology 1994;80:77-92.

11 Epstein $\mathrm{RH}$, Dexter F. Mediated interruptions of anaesthesia providers using predictions of workload from anaesthesia information management system data. Anaesth Intensive Care 2012;40:803-12.

12 Schmutz J, Hoffmann F, Heimberg E, et al. Effective coordination in medical emergency teams: the moderating role of task type. Eur $J$ Work Organ Psychol 2015;24:761-76.

13 Saadat H, Escobar A, Davis EA, et al. Task analysis of the preincision period in a pediatric operating suite: an independent observer-based study of 656 cases. Anesth Analg 2006;103:928-31.

14 Escobar A, Davis EA, Ehrenwerth J, et al. Task analysis of the preincision surgical period: an independent observer-based study of 1558 cases. Anesth Analg 2006;103:922-7.

15 Carson-Stevens A, Donaldson L, Sheikh A. The Rise of Patient Safety-II: Should We Give Up Hope on Safety-I and Extracting Value From Patient Safety Incidents? Comment on "False Dawns and New Horizons in Patient Safety Research and Practice". Int J Health Policy Manag 2018;7:667-70.

16 Braithwaite Jet al. Complexity science in healthcare aspirations, approaches, applications and accomplishments. A White paper. (Australian Institute of Health Innovation, Macquarie University 2017.

17 Hollnagel E. Safety-I and safety-II: the past and future of safety management. 1. Ashgate Publishing Ltd, 2014.

18 Iflaifel M, Lim RH, Ryan K, et al. Resilient health care: a systematic review of conceptualisations, study methods and factors that develop resilience. BMC Health Serv Res 2020;20:1-49.

19 Douglas HE, Raban MZ, Walter SR, et al. Improving our understanding of multi-tasking in healthcare: drawing together the cognitive psychology and healthcare literature. App/ Ergon 2017;59:45-55.

20 Chisholm CD, Collison EK, Nelson DR, et al. Emergency department workplace interruptions: are emergency physicians "interrupt-driven" and "multitasking"? Acad Emerg Med 2000;7:1239-43.

21 Forsberg HH, Muntlin Athlin Åsa, von Thiele Schwarz U. Nurses' perceptions of multitasking in the emergency department: effective, fun and unproblematic (at least for me) - a qualitative study. Int Emerg Nurs 2015;23:59-64.

22 Berg LM, Florin J, Ehrenberg A, et al. Reasons for interrupting colleagues during emergency department work - A qualitative study. Int Emerg Nurs 2016;29:21-6.

23 Laxmisan A, Hakimzada F, Sayan OR, et al. The multitasking clinician: decision-making and cognitive demand during and afte team handoffs in emergency care. Int J Med Inform 2007;76:801-11.

24 Westbrook JI, Raban MZ, Walter SR, et al. Task errors by emergency physicians are associated with interruptions, multitasking, fatigue and working memory capacity: a prospective, direct observation study. BMJ Qual Saf 2018;27:655-63.

25 Li L, Hains I, Hordern T, et al. What do ICU doctors do? A multisite time and motion study of the clinical work patterns of registrars. Crit Care Resusc 2015;17:159-66.
26 Bellandi T, Cerri A, Carreras G, et al. Interruptions and multitasking in surgery: a multicentre observational study of the daily work patterns of doctors and nurses. Ergonomics 2018;61:40-7.

27 Göras C, Olin K, Unbeck M, et al. Tasks, multitasking and interruptions among the surgical team in an operating room: a prospective observational study. BMJ Open 2019;9:1-12.

28 Schiff JH, Welker A, Fohr B, et al. Major incidents and complications in otherwise healthy patients undergoing elective procedures: results based on 1.37 million anaesthetic procedures. $\mathrm{Br} \mathrm{J}$ Anaesth 2014;113:109-21.

29 Al-Hakim L, Arora S, Sevdalis N. Impact of disruptions on anaesthetic workflow during anaesthesia induction and patient positioning: a prospective study. Eur J Anaesthesiol 2016;33:581-7.

30 Weinger MB. Human factors research in anesthesia patient safety: techniques to elucidate factors affecting clinical task performance and decision making. J. Am. Med. Informatics Assoc 2002;9:58S-63.

31 Mcmullan RD, Urwin R, Gates $\mathrm{P}$, et al. Are operating room distractions, interruptions and disruptions associated with performance and patient safety? A systematic review and metaanalysis. Int J Qual Health Care 2021;33:1-10.

32 Savoldelli GL, Thieblemont J, Clergue F, et al. Incidence and impact of distracting events during induction of general anaesthesia for urgent surgical cases. Eur J Anaesthesiol 2010;27:683-9.

33 Walter SR, Dunsmuir WTM, Westbrook Jl. Studying interruptions and multitasking in situ: the untapped potential of quantitative observational studies. Int J Hum Comput Stud 2015;79:118-25.

34 Antoniadis S, Passauer-Baierl S, Baschnegger H, et al. Identification and interference of intraoperative distractions and interruptions in operating rooms. J Surg Res 2014;188:21-9.

35 Cohen TN, Cabrera JS, Sisk OD, et al. Identifying workflow disruptions in the cardiovascular operating room. Anaesthesia 2016;71:948-54.

36 Morgan L, Robertson E, Hadi M, et al. Capturing intraoperative process deviations using a direct observational approach: the glitch method. BMJ Open 2013;3:e003519.

37 Boquet Aet al. A theoretical model of flow disruptions for the anesthesia team during cardiovascular surgery. J. Patient Saf 2017;00:1-6.

38 Campbell G, Arfanis K, Smith AF. Distraction and interruption in anaesthetic practice. Br J Anaesth 2012;109:707-15.

39 Jenkins A, Wilkinson JV, Akeroyd MA, et al. Distractions during critical phases of anaesthesia for caesarean section: an observational study. Anaesthesia 2015;70:543-8.

40 Crockett CJ, Donahue BS, Vandivier DC. Distraction-Free induction zone. Anesth. Analg 2018;129:1.

41 Broom MA, Capek AL, Carachi P, et al. Critical phase distractions in anaesthesia and the sterile cockpit concept. Anaesthesia 2011;66:175-9.

42 Slagle JM, Porterfield ES, Lorinc AN, et al. Prevalence of potentially distracting Noncare activities and their effects on vigilance, workload, and nonroutine events during anesthesia care. Anesthesiology 2018;128:44-54.

43 Meeusen V, van Zundert A, Hoekman J, et al. Composition of the anaesthesia team: a European survey. Eur J Anaesthesiol 2010;27:773-9.

44 Flin R, Maran N. Basic concepts for Crew resource management and non-technical skills. Best Pract Res Clin Anaesthesiol 2015;29:27-39.

45 Lyk-Jensen HT, Jepsen RMHG, Spanager L, et al. Assessing nurse anaesthetists' non-technical skills in the operating room. Acta Anaesthesiol Scand 2014;58:794-801.

46 Rönnberg L, Nilsson U, Hellzén O, et al. The art is to Extubate, not to Intubate-Swedish registered nurse Anesthetists' experiences of the process of extubation after general anesthesia. J Perianesth Nurs 2019;34:789-800.

47 Göras C, Nilsson U, Ekstedt M, et al. Managing complexity in the operating room: a group interview study. BMC Health Serv Res 2020;20:1-12.

48 Henrichs BM, Avidan MS, Murray DJ, et al. Performance of certified registered nurse anesthetists and anesthesiologists in a simulationbased skills assessment. Anesth Analg 2009;108:255-62.

49 Rönnberg L, Nilsson U. Swedish Nurse Anesthetists' Experiences of the WHO Surgical Safety Checklist. J. Perianesthesia Nurs 2015;30:468-75.

50 Sundqvist A-S, Nilsson U, Holmefur M, et al. Promoting personcentred care in the perioperative setting through patient advocacy: an observational study. J Clin Nurs 2018;27:2403-15.

51 Sundqvist A-S, Anderzén-Carlsson A, Nilsson U, et al. Protective Nursing Advocacy: Translation and Psychometric Evaluation of an Instrument and a Descriptive Study of Swedish Registered Nurse Anesthetists' Beliefs and Actions. J. Perianesthesia Nurs 2018;33:58-68. 
52 Nordström A, Wihlborg M. A phenomenographic study of Swedish nurse Anesthetists' and or nurses' work experiences. Aorn J 2019;109:217-26.

53 Riksföreningen för anestesi och intensivvard. Description of competence for registered nurse with graduate diploma in specialist nursing-anaesthesia care 2012.

54 WMA. Wma Declaration of Helsinki- ethical principles. World Medical Association 2013:29-32.

55 Westbrook Jl, Ampt A. Design, application and testing of the work observation method by activity timing (WOMBAT) to measure clinicians' patterns of work and communication. Int J Med Inform 2009;78:S25-33.

56 Grundgeiger T, Dekker S, Sanderson P, et al. Obstacles to research on the effects of interruptions in healthcare. BMJ Qual Saf 2016;25:392-5.

57 Fleiss JL, Levin B, Paik MC. Statistical methods for rates and proportions. 3. Wiley, 2003.

58 Rivera AJ. A socio-technical systems approach to studying interruptions: understanding the interrupter's perspective. Appl Ergon 2014;45:747-56.

59 Kolbe M, Grote G, Waller MJ, et al. Monitoring and talking to the room: autochthonous coordination patterns in team interaction and performance. J Appl Psychol 2014;99:1254-67.

60 Burtscher MJ, Wacker J, Grote G, et al. Managing nonroutine events in anesthesia: the role of adaptive coordination. Hum Factors 2010;52:282-94.
61 Wunder LL. Effect of a Nontechnical skills intervention on first-year student registered nurse Anesthetists' skills during crisis simulation. Aana J 2016;84:46-51.

62 Zala-Mezö E, Wacker J, Künzle B, et al. The influence of standardisation and task load on team coordination patterns during anaesthesia inductions. Quality and Safety in Health Care 2009;18:127-30.

63 Anderson JE, Ross AJ, Back J, et al. Beyond 'find and fix': improving quality and safety through resilient healthcare systems. Int J Qual Heal care J Int Soc Qual Heal Care 2020;32:204-11.

64 Broom MA, Capek AL, Carachi P, et al. Critical phase distractions in anaesthesia and the sterile cockpit concept. Anaesthesia 2011;66:175-9.

65 Westbrook Jl. Interruptions to clinical work: how frequent is too frequent? J Grad Med Educ 2013;5:337-9.

66 Bergström J, van Winsen R, Henriqson E. On the rationale of resilience in the domain of safety: a literature review. Reliab Eng Syst Saf 2015;141:131-41.

67 Skaugset LM, Farrell S, Carney M, et al. Can you Multitask? evidence and limitations of task switching and multitasking in emergency medicine. Ann Emerg Med 2016;68:189-95.

68 Dixon-Woods M. Why is patient safety so hard? a selective review of ethnographic studies. J Health Serv Res Policy 2010;15:11-16. 